\title{
FLUXO DE LÍQUIDO NUM MOLDE DE BEAM BLANK E A REMOÇÃO DE INCLUSÕES*
}

\author{
Weslei Viana Gabriel ${ }^{1}$ \\ Johne Jesus Mol Peixoto ${ }^{1}$ \\ Gesiane Letícia Alves ${ }^{2}$ \\ Carlos Antonio da Silva ${ }^{3}$ \\ Itavahn Alves da Silva ${ }^{3}$ \\ Varadarajan Seshadri ${ }^{4}$
}

\section{Resumo}

Por meio do uso de técnicas de modelamento físico e matemático, caracterizou-se o fluxo de líquido, no interior de um molde de beam blank, quando alimentado por apenas uma válvula submersa constituída por três portas de saída cilíndricas e espaçadas de $120^{\circ}$. Verificou-se que o fluxo do jato de líquido que atravessa a alma se espalha ao longo de toda a superfície do flange antes de se recircular na região central do molde. Através da técnica de injeção de traçador e análise do perfil de velocidade, percebe-se que os jatos direcionados para as quinas do flange recirculam rapidamente e, por isto, se espalham numa pequena região. Este tipo de válvula se caracteriza também por ter uma maior intensidade de oscilação da superfície do que a válvula do tipo tubular. Foram realizados testes para simular a taxa de remoção de inclusões. Os dados obtidos mostram que esta válvula é mais eficiente na limpidez do aço do que uma válvula tubular simples.

Palavras-chave: Lingotamento Contínuo; Válvula Submersa; Beam Blank; Fluxo.

\section{FLUID FLOW INSIDE A BEAM BLANK MOLD AND INCLUSIONS REMOVAL Abstract}

The flow field inside a beam blank mold fed with only one submerged entry nozzle with three cylindrical ports spaced of $120^{\circ}$ has been described by physical and mathematical modeling. It has been verified that fluid coming from the port feeding the web spreads itself at the opposite flange surface before recirculating in the center of the mold. Tracer injection technique and analysis of the velocity profile show that the jets directed to the flange tips recirculate quickly and then spread in a smaller region. This type of SEN is characterized by a higher intensity of oscillation at the free surface than a straight nozzle used for comparison purposes. The rate of removal of inclusions inside the mold has been evaluated. The data show that this SEN is more efficient in regards to steel cleanness than a straight through nozzle.

Keywords: Continuous Casting; SEN; Beam Blank; Flow.

1 Engenheiro Metalurgista, Doutorando em Engenharia de Materiais/REDEMAT, Universidade Federal de Ouro Preto, Minas Gerais Brasil.

2 Graduando em Metalurgia, Escola de Minas, Ouro Preto, Minas Gerais, Brasil..

3 Engenheiro Metalurgista, Ph.D., Professor, Universidade Federal de Ouro Preto, Escola de Minas, Ouro Preto, Minas Gerais, Brasil..

4 Engenheiro Metalurgista, Dr.Ing., Professor Emérito, Universidade Federal de Minas Gerais, Belo Horizonte, Minas Gerais, Brasil. 


\section{INTRODUÇÃO}

Os parâmetros operacionais (velocidade de lingotamento, profundidade de imersão da válvula submersa), qualidade e consumo específico do pó fluxante, fluxo térmico e campo de velocidades no aço líquido são alguns dos fatores que influenciam na qualidade do produto de lingotamento contínuo. Além disto, em moldes de beam blank, a complexidade da geometria torna ainda mais desafiador o controle de qualidade do produto lingotado. A utilização de duas válvulas submersas propicia a reduzir a assimetria do fluxo de fluido ao longo do molde (Peixoto et al. [1]). Porém esta configuração traz dificuldades operacionais e até mesmo de projeto do sistema de alimentação (De Santis et al. [2]).

Entender as características de fluxo geradas pelo sistema de alimentação adotado em moldes de beam blank é importante para a definição das melhores condições operacionais, visando maior produtividade, maior limpidez do aço e segurança operacional (Beaton et al. [3]). Xuo e Zhu [4] relatam que a utilização de válvulas tubulares resulta numa superfície livre praticamente inativa, o que dificulta a fusão do pó fluxante e prejudica a lubrificação das paredes do molde. Já a utilização de duas válvulas com portas laterais na saída permite desenvolver um fluxo de fluido e de calor mais uniforme gerando uma casca solidificada de espessura mais homogênea, além de intensificar a oscilação superficial. Chen et al. [5] observaram redução na profundidade de impacto do jato de líquido oriundo deste modelo de válvula, facilitando a remoção de inclusões não metálicas. Onishi et al. [6] demonstraram que a limpidez do aço aumenta quando se substitui válvulas tubulares por válvulas com portas laterais.

Zhang et al. [7] caracterizaram o fluxo de fluido no interior do molde de beam blank para o caso de jato aberto. Neste caso, observa-se um grande arraste de bolhas de ar para o interior do molde e alteração significativa no perfil de velocidade ao longo do molde. A profundidade de imersão do funil tem grande influência sobre a profundidade de impacto das bolhas e da oscilação da superfície livre.

A configuração das portas de saída também exerce importante influência sobre as características de fluxo. Najjar et al. [8] estudaram a influência do ângulo de inclinação das portas, formato e espessura das paredes das portas sobre as características do jato de líquido, mostrando que todos estes fatores afetam a inclinação do jato de líquido e em consequência o comportamento da superfície do banho. Hibbeler et al. [9] demostraram que a inclinação do jato de líquido é um dos fatores que impactam na possibilidade de entranhamento de escória.

Finalmente, este trabalho tem como objetivo descrever o fluxo de fluido, bem como avaliar a oscilação da superfície livre e a taxa de remoção de inclusões em molde de beam blank alimentado por apenas uma válvula com três portas laterais na saída.

\section{MATERIAIS E MÉTODOS}

Os testes foram realizados utilizando um modelo construído em acrílico, escala 1:1 de um molde de beam blank com dimensões $499 \mathrm{mmX} 415 \mathrm{mmX} 125 \mathrm{~mm}$ e $1,5 \mathrm{~m}$ de altura, utilizando como fluido a água líquida em temperatura ambiente.

Para a análise qualitativa do fluxo acompanhou-se o percurso de um traçador ao longo do molde após o mesmo ser injetado na entrada da válvula.

Mensurou-se a oscilação da superfície livre através de um conjunto de sensores ultrassônicos modelo SICK UM30-21_118. As medições deste equipamento são pontuais e os pontos escolhidos para análise estão destacados na figura 1b). 
O modelo de válvula proposto (Figura 1(a)) possui três portas laterais na saída espaçados entre si de $120^{\circ}$ : duas portas com menor diâmetro $(16 \mathrm{~mm})$ e outra com maior diâmetro $(47 \mathrm{~mm})$ que é posicionada em direção à alma. A porta maior possui um deslocamento de $20 \mathrm{~mm}$ com relação à base da válvula. A profundidade de imersão é mensurada pela distância a partir da borda superior desta saída até a superfície livre. E os valores de profundidade de imersão utilizados foram de $200 \mathrm{~mm}$ e $250 \mathrm{~mm}$. O ângulo de inclinação das portas $(\Theta)$ foi de $-5^{\circ}$. Sendo que na análise de inclusões comparou-se os dados desta válvula com a válvula de $+5^{\circ}$.

Para simular o fluxo de inclusões no molde injetou-se $5 \mathrm{~g}$ de partículas com granulometria entre $100 \mu \mathrm{m}$ e $200 \mu \mathrm{m}$ na entrada da válvula submersa. Coletando-as na saída durante o período de 5 minutos. Após secagem, as particulas coletadas foram pesadas. A taxa de flotação de inclusões é dada pela diferença entre o coletado e o total injetado.

Considerando que a viscosidade cinemática da água à temperatura ambiente e a do aço a $1600^{\circ} \mathrm{C}$ são iguais, é possível determinar a seguinte equação que correlaciona o tamanho das partículas no modelo com as inclusões no equipamento industrial:

$$
\frac{r_{p, M}}{r_{p, P}}=\lambda^{\frac{1}{4}}\left(\frac{1-\frac{\rho_{p, M}}{\rho_{L, M}}}{1-\frac{\rho_{p, P}}{\rho_{L, P}}}\right)^{\frac{1}{2}}
$$

Onde: $r_{p, M}$ e $r_{p, P}$ são os raios da inclusão no modelo e no protótipo respectivamente. $\rho_{L, P}, \rho_{L, M}, \rho_{p, M}$ e $\rho_{p, P}$ são as densidades do aço, da água, da inclusão no modelo e no protótipo respectivamente.

Tabela 1 Propriedades do aço e da água consideradas neste teste:

\begin{tabular}{l|c|c}
\multicolumn{1}{c|}{ Propriedades } & Industrial & Modelo \\
\hline Densidade do líquido $\mathrm{kg} / \mathrm{m}^{3}$ & 7000 & 1000 \\
Densidade da partícula & 3000 & 900 \\
$\mathrm{~kg} / \mathrm{m}^{3}$ & &
\end{tabular}

Para um fator de escala $(\lambda)$ igual a 1 e considerando os dados da tabela 1 , a expressão anterior sugere que partículas com diâmetro entre $100 \mu \mathrm{m}$ e $200 \mu \mathrm{m}$, equivalem a inclusões no banho metálico na faixa granulométrica entre $41,84 \mu \mathrm{m}$ e 83,68 $\mu \mathrm{m}$.

Para complementar a descrição do fluxo foram realizadas também simulação computacional em regime permanente utilizando o software Ansys CFX versão 17.1. Considerou-se a água um fluido newtoniano, temperatura constante e igual a $25^{\circ} \mathrm{C}$. O modelo aplicado foi o $\mathrm{k}-\epsilon$, no qual se resolve as equações da continuidade, de Navier-Stokes e da viscosidade efetiva, além das equações auxiliares para determinação de $k$ (energia de turbulência) e $€$ (taxa de dissipação de energia cinética).

Condições de contorno aplicáveis ao problema:

- Paredes - Condição de não deslizamento nas paredes do molde e da válvula;

- Condição de livre deslizamento no menisco; 
- Vazão mássica na entrada - 1,667kg/s; 2,083kg/s e 2,5kg/s para as velocidades de lingotamento equivalentes a $0,78 \mathrm{~m} / \mathrm{min} ; 0,98 \mathrm{~m} / \mathrm{min}$ e $1,2 \mathrm{~m} / \mathrm{min}$ respectivamente.

Os planos utilizados na análise dos perfis de velocidade para caracterização do fluxo via CFD estão representados na figura 1(b) pelas linhas $A A$ e BB, que representam o plano de simetria e o plano que liga a porta de menor diâmetro à quina do flange respectivamente. Já a linha BC representa de maneira esquemática a direção da linha central do jato de líquido. Nesta direção avaliou-se a velocidade do fluido.

Para análise de oscilação superficial, bem como de dispersão de traçador no fluido, realizaram-se simulações computacionais em regime transiente, timestep de 0,005 e tempo total de 30 s.

a)

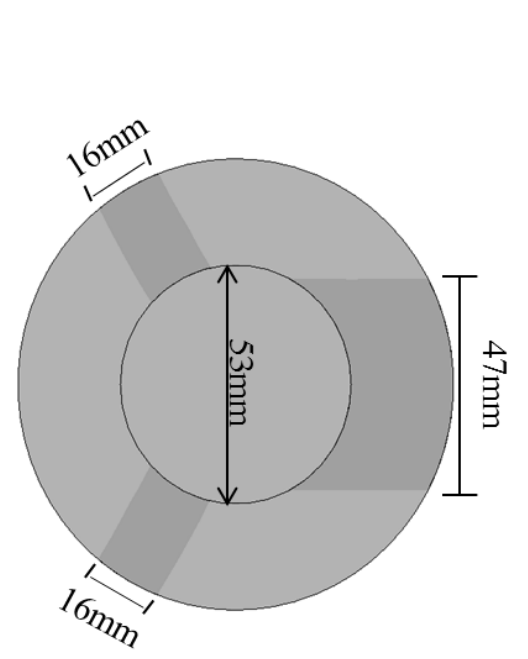

b)

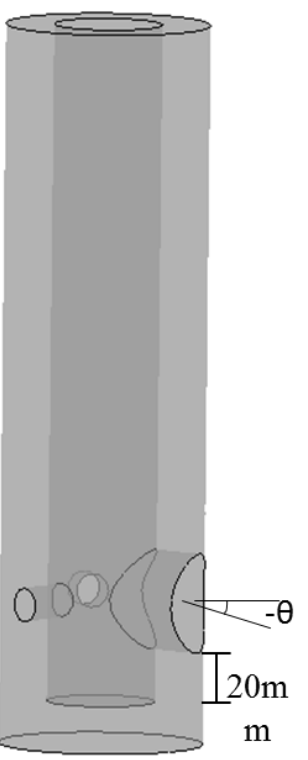

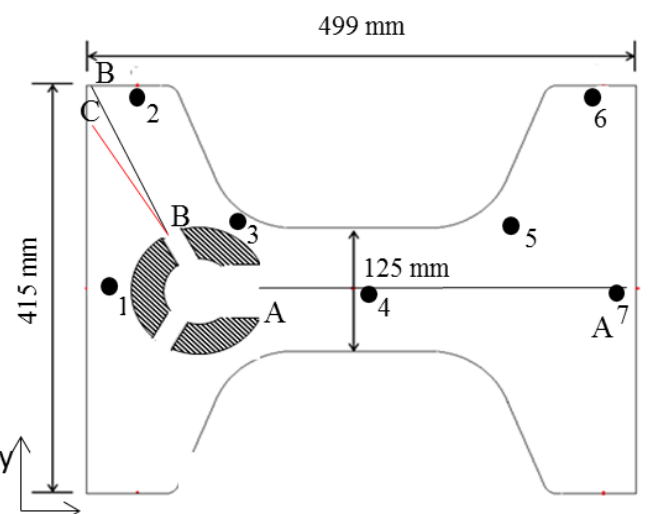

$\mathrm{x}$

Figura 1 Modelo esquemático da válvula e do molde de beam blank. a) seção transversal e vista isométrica da válvula. b) modelo esquemático do molde de beam blank.

\section{RESULTADOS E DISCUSSÃO}

\subsection{Análise de Fluxo}

A figura 2 descreve o fluxo de fluido no molde de beam blank alimentado pela válvula com portas laterais através da dispersão de um traçador para diferentes velocidades de lingotamento. Pode-se verificar que o jato de líquido direcionado para a alma se espalha ao atingir a superfície do flange oposto, sendo que uma parcela do mesmo segue em direção à superfície livre enquanto o restante do fluido se desloca para baixo formando uma zona de recirculação a aproximadamente $600 \mathrm{~mm}$ do menisco. Além disto, uma parcela deste fluido atinge rapidamente a saída do molde (considerando a altura do molde de $800 \mathrm{~mm}$ ). Esta característica pode facilitar o aprisionamento de inclusões presente no aço. O forte espalhamento do fluido no flange oposto implica em lenta recirculação de fluido na região inferior. 
O jato de líquido oriundo das portas de menor diâmetro atinge as quinas do flange próximo à válvula e em seguida se espalha ao longo da superfície e do filete. $\mathrm{A}$ porção que atravessa o filete encontra com o fluxo ascendente do jato de líquido que atravessa a alma e ambos descem acompanhando o jato de líquido que atravessa a alma.

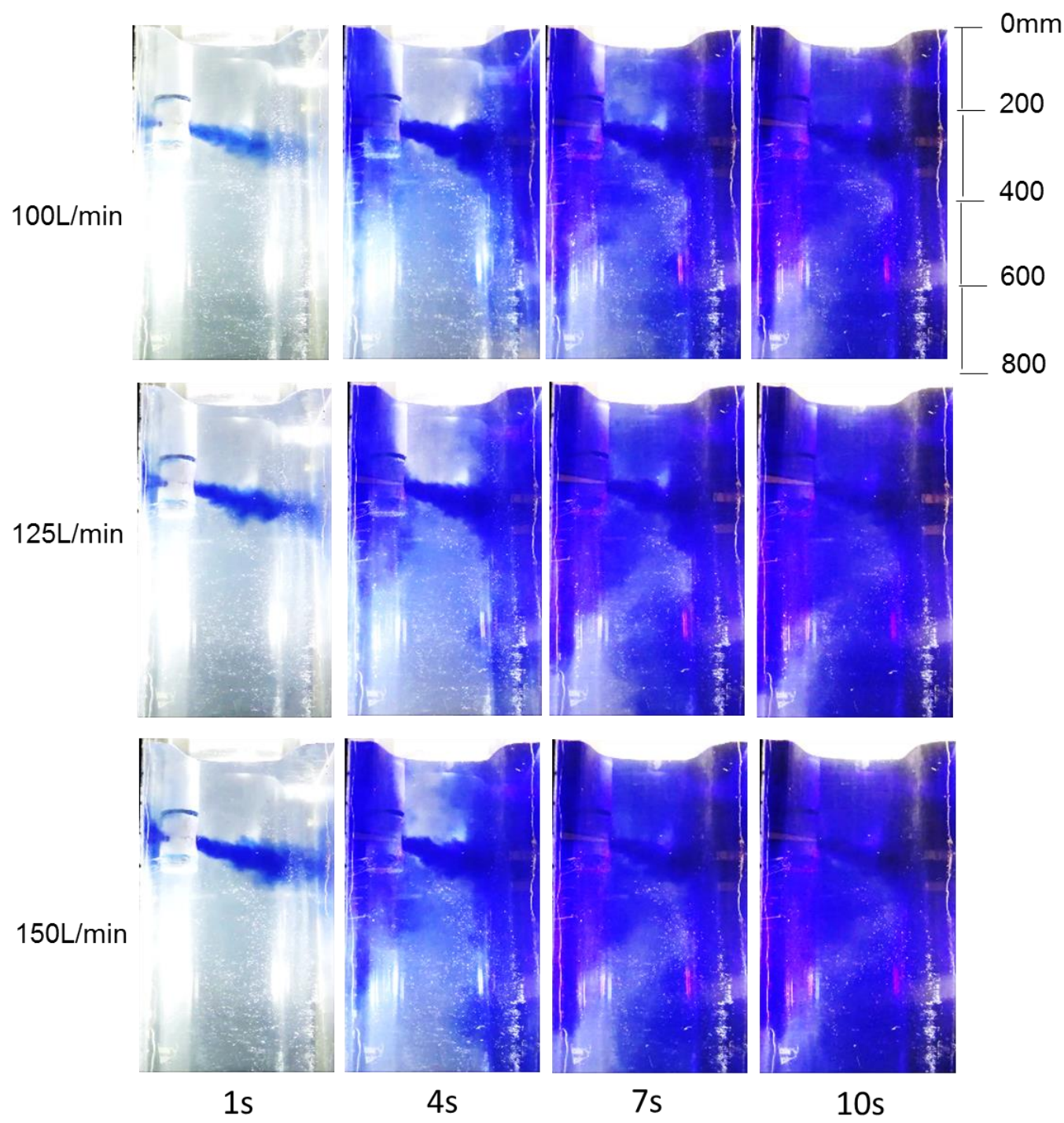

Figura 2 Análise do fluxo através da injeção de traçador para diferentes vazões.

Simulações matemáticas em condições de regime transiente para a dispersão de traçador também permitem avaliar o comportamento do fluido, sendo os resultados semelhantes aos obtidos via modelagem física, conforme figura 3. 

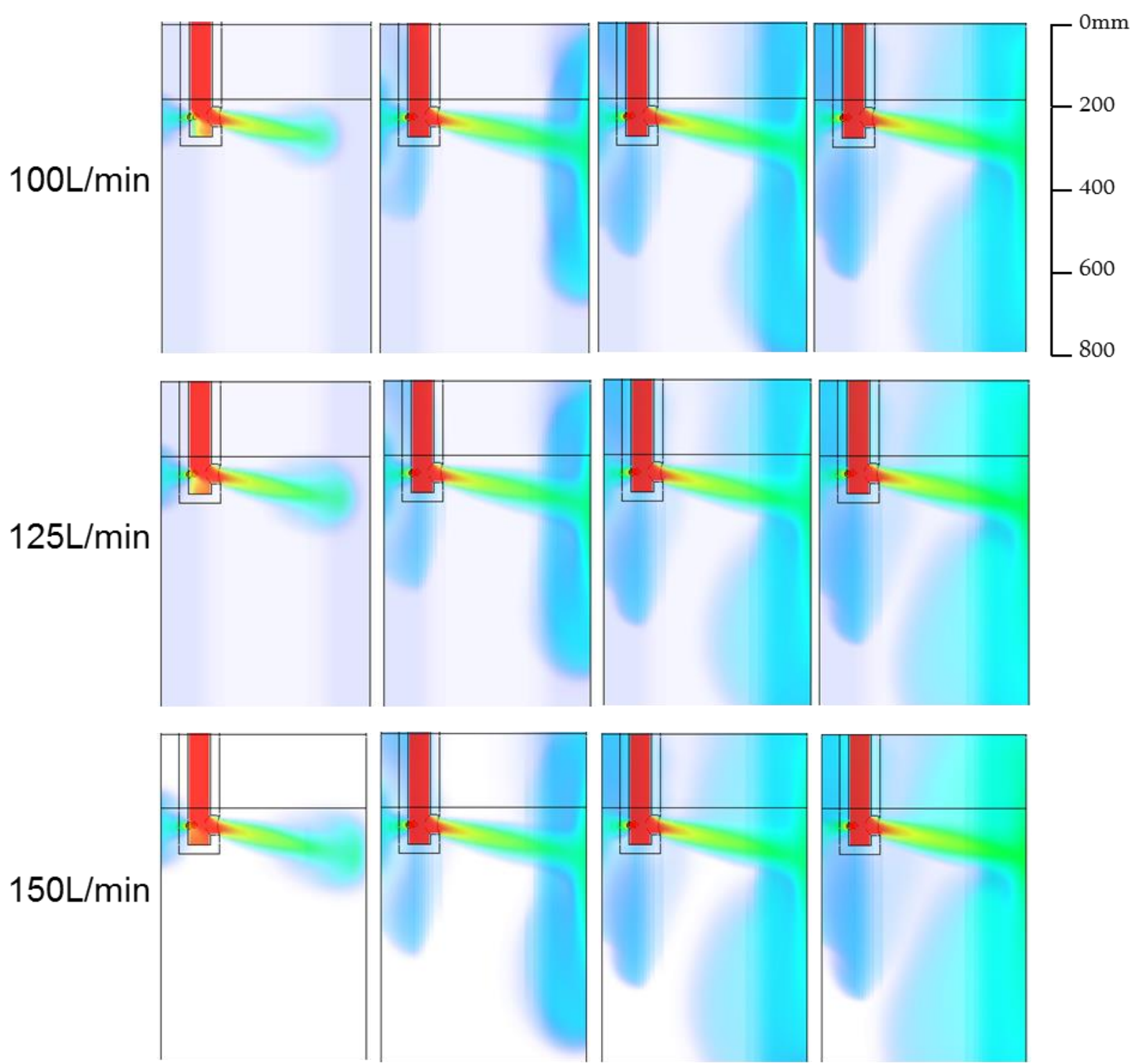

1s
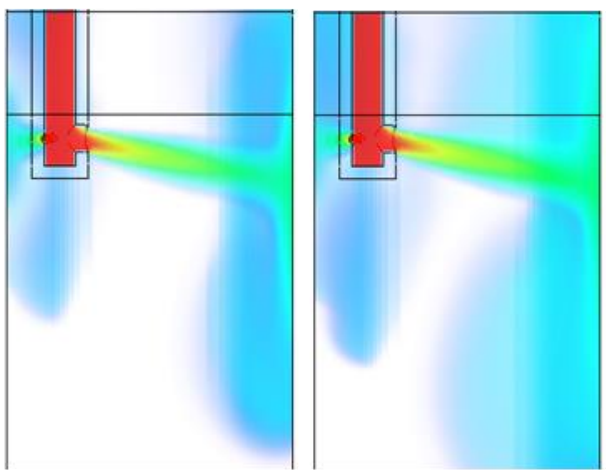

$7 \mathrm{~s}$

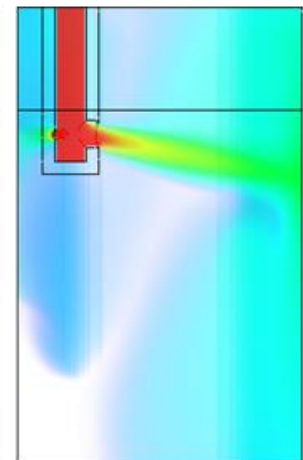

$10 \mathrm{~s}$

Figura 3 Análise do fluxo através da injeção de traçador para diferentes vazões, via CFD.

A figura 4 apresenta o perfil de velocidade ao longo dos planos AA e BB para deferentes vazões. As alterações na velocidade de lingotamento não implicam em alterações significativas do perfil de velocidade. No plano AA observa a formação de um vórtice próximo a superfície livre em função da recirculação do fluido ascendente. Na região inferior do molde também é observada uma zona de recirculação próxima à saída do molde.

O perfil de velocidade no plano BB permite analisar que o jato de líquido oriundo das portas de menor diâmetro ao atingir a superfície se espalha, gerando dois vórtices. Implicando em rápido preenchimento da região conforme destacado nas figuras $2 \mathrm{e}$ 3. Os jatos de líquido oriundos das portas menores alimentam principalmente a região superior do flange em que a válvula está posicionada. 


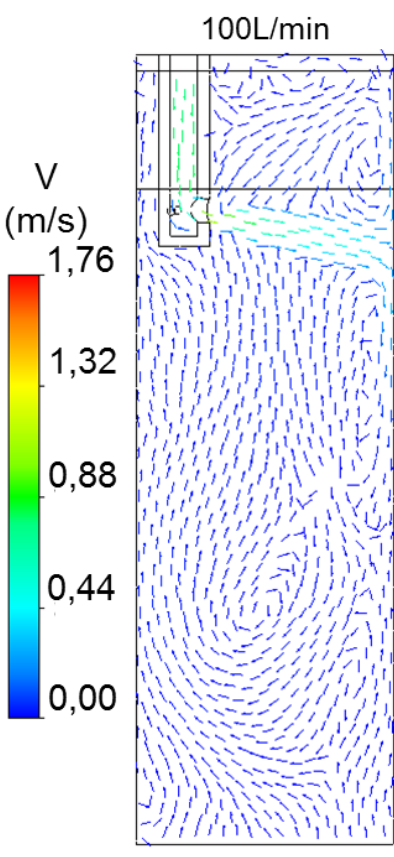

a)

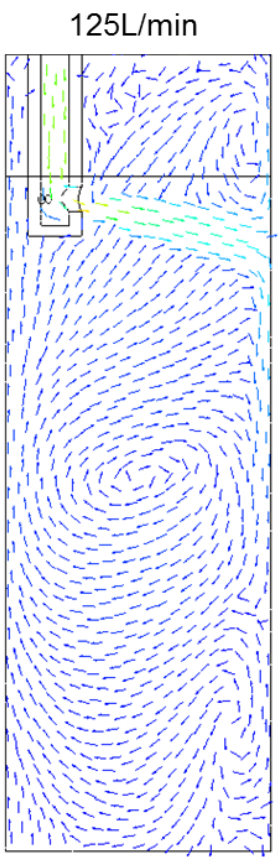

b)

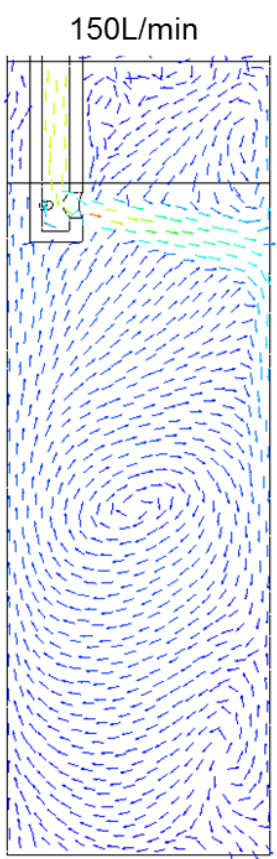

c)

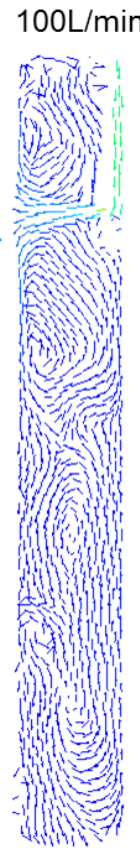

d)

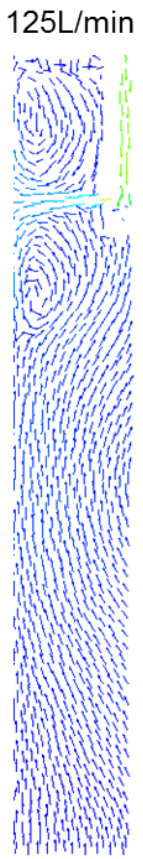

e)

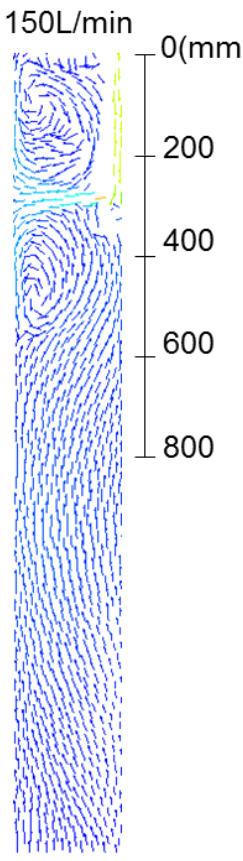

f)

Figura 4 Análise do perfil de velocidade em função da vazão. a), b) e c) Plano AA. c), d e e) plano BB.

A figura 5 apresenta o perfil de velocidade em um plano que corta o centro das portas de saída. O jato de líquido que evade da porta de menor diâmetro impinge na superfície do flange, e em seguida segue em direção à quina do mesmo onde recircula e vai ao encontro do jato principal. O centro do flange ao lado da válvula se caracteriza por ter um fluxo ascendente e ser uma região de menor velocidade nesta seção.

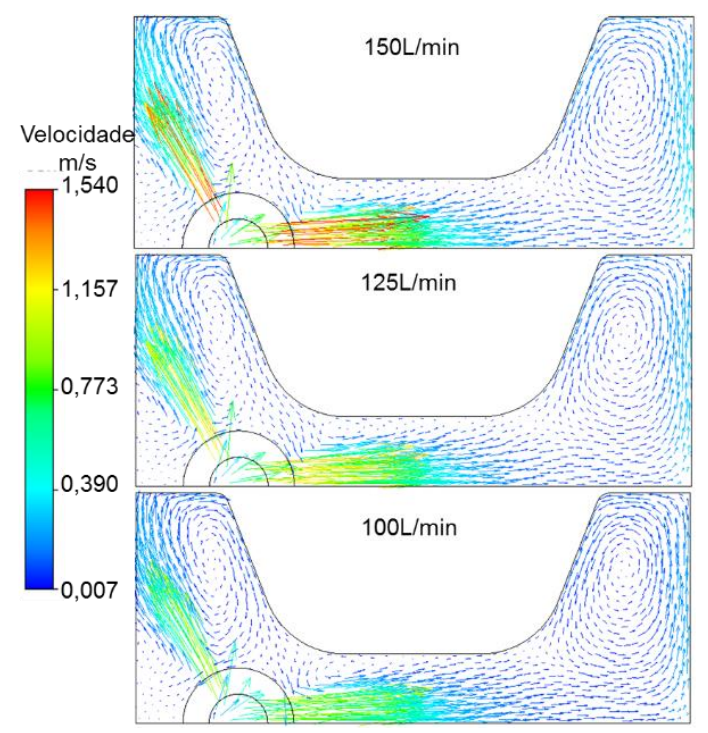

Figura 5 Perfil de velocidade na seção transversal - plano no centro das portas de saída.

A figura 6 compara as velocidades no centro dos jatos de líquido por meio de dados obtidos via CFD. A velocidade do jato de líquido na saída das portas menores é cerca de $10 \%$ inferior à da porta direcionada para a região da alma. O gradiente de velocidade no jato de líquido oriundo da porta menor é superior em função da pequena distância da superfície do flange em que a válvula foi posicionada. Já o jato 
de líquido da porta maior atravessa a alma antes de atingir a superfície do flange oposto à mesma. Gabriel et al. [10] demonstraram que os valores de velocidade obtidos pela técnica CFD (utilizando o setup descrito neste trabalho) são corroborados pelos dados obtidos por simulação física através da técnica PIV.
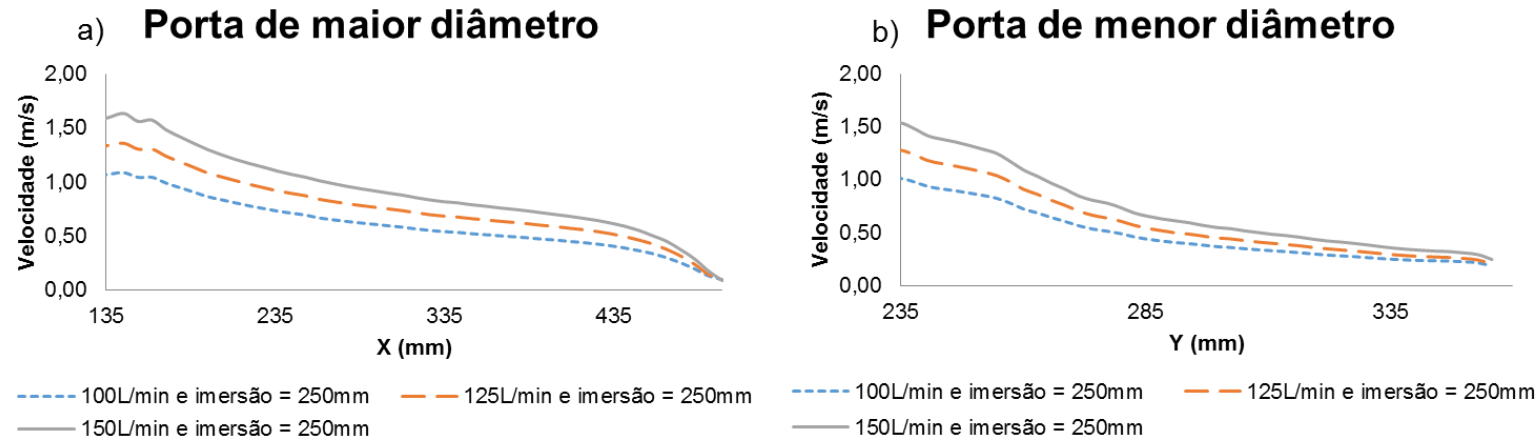

Figura 6 Valores de velocidade no centro do jato. a) porta de maior diâmetro. b) porta de menor diâmetro.

\subsection{Oscilação Superficial}

Outra característica do fluxo para a válvula com portas laterais é o aumento da flutuação superficial. A figura 7 apresenta o comportamento da superfície, obtido por filmagem do modelo físico e através de modelamento matemático respectivamente. Percebe-se que a região de maior turbulência encontra-se próximo ao flange oposto.

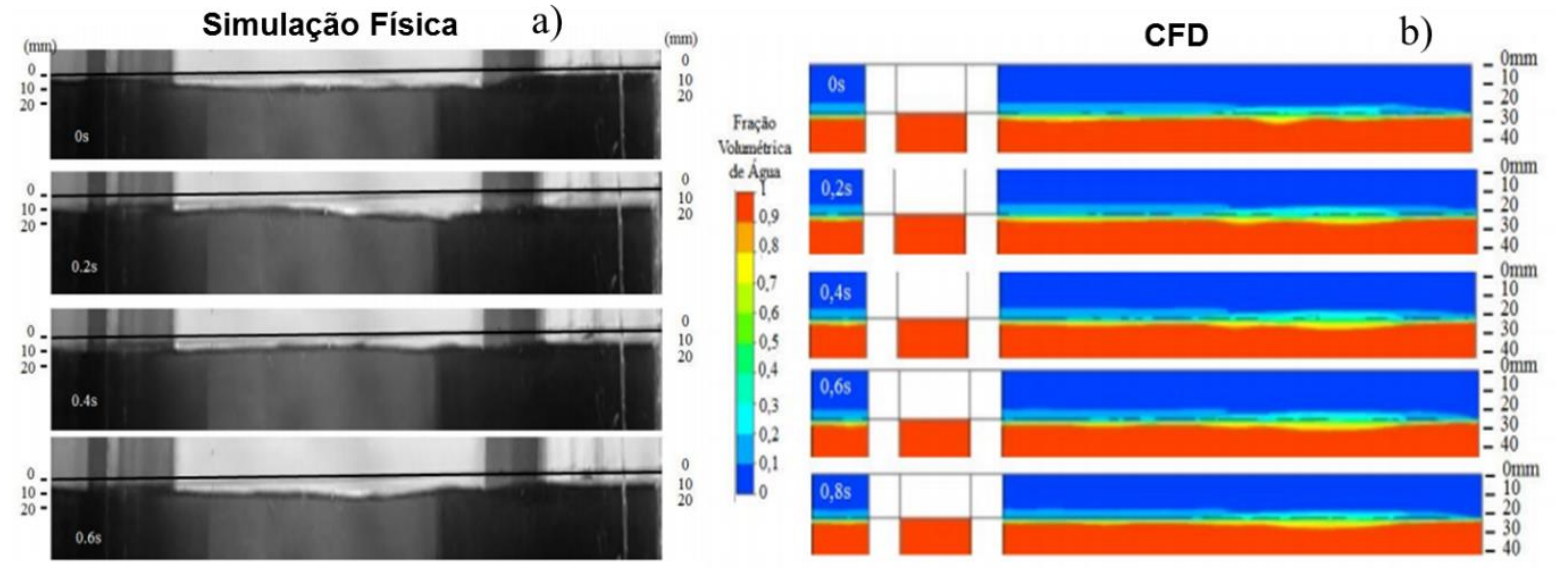

Figura 7 Análise da oscilação da superfície livre utilizando válvula com $-5^{\circ}$, vazão $=150 \mathrm{~L} / \mathrm{min}$ e profundidade de imersão $=200 \mathrm{~mm}$. a) modelagem física. b) análise da variação da fração volumétrica no plano de simétrica - CFD.

A figura 8a) apresenta valores de intensidade de oscilação da superfície livre da poça de líquido para a válvula com portas laterais da válvula submersa. $O$ aumento da vazão de lingotamento implica em aumento da intensidade de flutuação. A figura 8b) compara a intensidade de flutuação da válvula com portas laterais e a válvula em formato de tubo, no centro da alma e no centro do flange oposto (P4 e P7 respectivamente). Para este tipo de válvula a flutuação da superfície livre da poça de líquido é bem inferior à encontrada para a válvula submersa com portas laterais.

Além disto, a intensidade de flutuação da superfície livre da poça de líquido, para o caso da válvula tubular, não varia com a alteração da velocidade de lingotamento. Uma baixa intensidade de flutuação da superfície livre da poça de líquido reduz a 
tendência de ocorrência de entranhamento, porém pode dificultar a fusão do pó fluxante, comprometendo a lubrificação das paredes do molde e consequentemente a qualidade tanto do produto quanto do processo.

Peixoto et al. [1] mostraram que a utilização de válvulas submersas do tipo tubular se caracterizam por aumento na profundidade de imersão do jato de líquido, lenta renovação de fluido no menisco e consequentemente baixa intensidade de oscilação do mesmo.

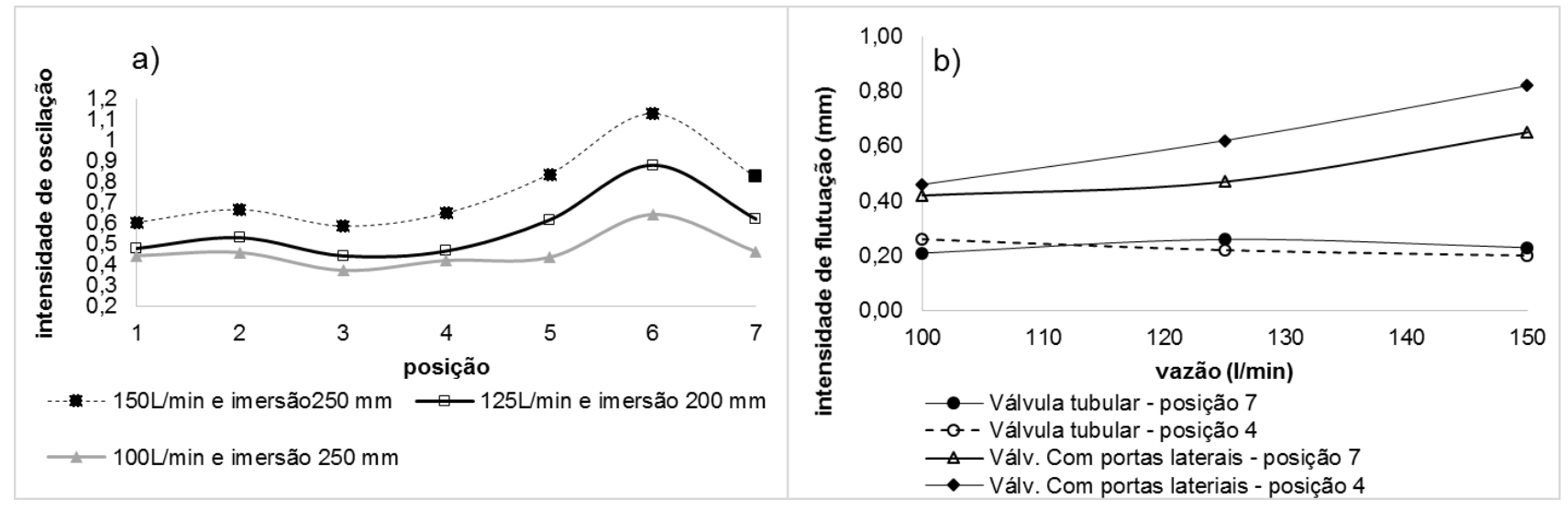

Figura 8 Valores de intensidade de oscilação da superfície livre. a) válvula com portas laterais. b) comparação entre a válvula tubular e a válvula com portas laterais.

\subsection{Remoção de inclusões}

A figura 9 apresenta um comparativo entre a influência da inclinação das portas de saída na percentagem de inclusões flotadas. Observa-se que quanto maior a inclinação das portas, maior a quantidade de inclusões flotadas. Este fator pode estar relacionado à redução na inclinação do jato, fato que facilitaria à inclusão atingir a superfície da poça de líquido na região do menisco. Gabriel et al. [10] observaram que a válvula com $+5^{\circ}$ produziu uma menor inclinação do jato.

$\mathrm{O}$ aumento da velocidade de lingotamento resulta em redução na percentagem de inclusões flotadas. Zhang et al [7] em testes realizados em molde de beam blank alimentado com duas válvulas submersas tubulares a uma velocidade de lingotamento de $1 \mathrm{~m} / \mathrm{min}$, encontraram taxa de remoção de inclusões de $25 \%$ e $27 \%$ para diâmetro interno da válvula com $30 \mathrm{~mm}$ e $40 \mathrm{~mm}$ respectivamente. Onishi et al. [6] observaram que a utilização de válvulas com saídas laterais aumenta a eficiência na remoção de inclusões em molde de beam blank. 


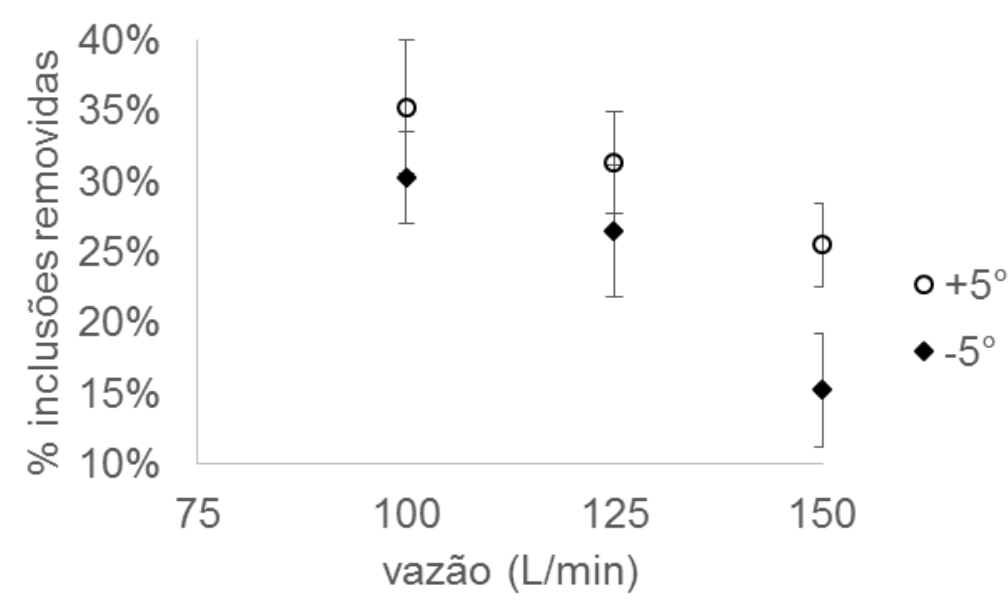

Figura 9 Influência da vazão e inclinação das portas de saída do fluido sobre a de remoção de

\section{CONCLUSÕES} inclusões.

Utilizando-se modelamento físico e matemático, a análise do comportamento do fluxo de fluido no interior do molde de lingotamento contínuo alimentado com apenas uma válvula submersa com três portas laterais resultou em:

- A recirculação de fluido inferior do molde é lenta devido ao espalhamento do jato de líquido no flange oposto.

- O jato de líquido oriundo das portas menores alimenta principalmente a região superior do flange em que a válvula submersa está posicionada. Este jato impinge na superfície do flange.

- A velocidade do jato de líquido na saída das portas de diâmetro inferior da válvula submersa corresponde à aproximadamente $90 \%$ da velocidade na porta de maior diâmetro; entretanto o gradiente à frente das portas de menor diâmetro é significativamente maior.

- A oscilação superficial no topo da poça (região do menisco) resultante para o modelo de válvula estudado é superior ao obtido para válvulas submersas tubulares.

- O aumento da velocidade de lingotamento implica em aumento na intensidade de oscilação superficial na região do menisco.

- A taxa de remoção de inclusões para este modelo de válvula submersa é superior ao encontrado para moldes de beam blank alimentados com duas válvulas submersas tubulares.

- A redução na velocidade de lingotamento e o aumento no ângulo de inclinação das portas de saída da válvula submersa aumentam a taxa de remoção de inclusões.

\section{Agradecimentos}

Os autores gostariam de agradecer FAPEMIG, à Coordenação de Aperfeiçoamento de Pessoal de Nível Superior (CAPES) pelo apoio financeiro, em especial pelas bolsas de pesquisa concedidas a W.V. Gabriel, G.L. Alves e J. J. M. Peixoto, respectivamente. 


\section{REFERÊNCIAS}

1 Peixoto, J.J.M., Gabriel, W.V., Ribeiro, L.Q, Silva, C.A, Silva, I.A, Seshadri, V. 2016. Computational and Physical Simulation of Fluid Flow Inside a Beam Blank Continuous Casting Mold. Journal of Materials Processing Technology. 233, 8999.

2 De Santis, M., Cristallini, A., Rinaldi, M., Sgro, A., 2014. Modelling-based Innovative Feeding Strategy for Beam Blanks Mould Casting Aimed at As-cast Surface Quality Improvement. ISIJ International. 54 (3), 496-503.

3 Beaton, J.W., Sgro, A., Burini, A., Razza, P., Azizola, Ali., 2015. Beam Blank In Submerged Pour Casting: Danieli Technology And Experience. METEC Second Estad, Dusseldorf, pp.1-6.

4 Xu, M., Zhu, M., 2015. Transport Phenomena in a Beam-Blank Continuous Casting Mold with Two Types of Submerged Entry Nozzle, ISIJ International, 55 (4), 791-798.

5 Chen, W., Zhang, Y.Z., Zhu, L.G., Zhang, C.J., Chen, Y., Wang, B.X., Wang, C., 2012. Three Dimensional FEM Study of Fluid Flow in Mould for Beam Blank Continuous Casting: Influence of Nozzle Structure and Parameters on Fluid Flow. Ironmaking and Steelmaking, 39 (8), 560-567.

6 Onishi, M., UEDA, T., Shinjo, Y., Mizota, H., Yao, M., Fujimura, T., 1981. Continuous Casting of Beam Blanks. Kawasaki Steel Technical Report, $N^{\circ} 3$, 13-25.

7 Zhang,L.,Cheng,D.Long,M.,Xie,X.,Zhang,X.,Ma,Y. Hydraulic Simulation of Fluid Flow in Beam Blank Continuos Casting Mold With Double Nozzles. EPD Congress 2014, 375-384.

8 Najjar, F.M., Thomas, B.G., Hershey, D.E., 1995. Numerical Study of Steady Turbulent Flow Through Bifurcated Nozzles in Continuous Casting. Metallurgical Transactions B. 26B (4), 749-765.

9 Hibbeler, L.C., Thomas, B., 2013. Mold Slag Entrainment Mechanisms in Continuous Casting Molds. Iron \& Steel Technology. 16, 121-136.

10 Gabriel, W.V., Peixoto, J.J.M., Silva, C.A, Silva, C.A, Silva, I.A, Seshadri, V. 2016. The Influence of the Geometry of a Three Orifice Nozzle on the Flow Field Inside a Beam Blank Mold. Journal of Materials Processing Technology. 245, 232-240. 